\title{
Isolation of Cryptococcus spp. and Histoplasma capsulatum from soil and Bird Droppings at Kulik Bird Sanctuary, Raiganj, West Bengal, India
}

\author{
Nibedita Haldar ${ }^{1 *}$, Atasi Maji ${ }^{2}$, Debaprasad Pal $^{3}$ and Atanu Roy ${ }^{4}$ \\ Department of Microbiology, MGM Medical College, LSK hospital, Kishanagar, Bihar, India
}

A B S T R A C T

\begin{tabular}{|l|}
\hline Ke y w o r d s \\
$\begin{array}{l}\text { Cryptococcus } \\
\text { neoformans, } \\
\text { Histoplasma } \\
\text { capsulatum, } \\
\text { Sabouraud's } \\
\text { dextrose agar. }\end{array}$ \\
\hline Article Info \\
\hline $\begin{array}{l}\text { Accepted: } \\
\text { 12January } 2017 \\
\text { Available Online: } \\
\text { 10 February } 2017\end{array}$ \\
\hline
\end{tabular}

Cryptococcosis is an acute, subacute or chronic fungal disease caused by an encapsulated Basidiomycetes yeast belonging to the genus Cryptococcus. Among the many species of the genus "Cryptococcus" ubiquitously present in the environment Cryptococcus neoformans and Cryptococcus gattii are clinically significant. Cryptococcal meningitis is considered as an AIDS defining condition and is the most common fungal infection of the central nervous system in patients with or without impaired immune function. Histoplasmosis is a systemic granulomatous disease caused by a dimorphic fungus, Histoplasma capsulatum. Clinical manifestations may be classified according to site (Pulmonary, extrapulmonary or disseminated), duration of infection (acute and chronic) and pattern of infection (primary Vs reactivation). Several studies have proved the association of these two pathogenic fungi with bird droppings and soil contaminated with bird droppings. Therefore the present study was undertaken over a period of one year (from $1^{\text {st }}$ Nov $2014-31^{\text {st }}$ Oct 2015) in the Department of Microbiology, Mata Gujri Memorial Medical College and Lions Seva Kendra Hospital, Kishanganj, Bihar with bird excreta samples and soil samples (fifty each) collected from Kulik Bird sanctuary, Raiganj, Uttar Dinajpur for isolation of these two fungi. After processing of the samples cultures were done on Sabouraud's dextrose agar with Chloramphenicol and $2^{\text {nd }}$ set of culture on SDA with chloramphenicol and cycloheximide. Both the culture sets were kept both at $25^{\circ} \mathrm{C}$ as well $37^{\circ} \mathrm{C}$ followed by Germ tube test, growth on corn meal agar, inositol assimilation test, nitrate assimilation test, growth on bird seed agar, growth at $37^{\circ} \mathrm{C}$ and urease test for $C$. neoformans and lactophonol cotton blue mount done to search thick walled large tuberculate macro conidia of $H$. capsulam from white or buff brown colonies on SDA at $25^{\circ} \mathrm{C}$. Fifty soil samples did not show any growth of $C$. neoformans whereas three out of fifty bird excreta samples showed growth of $C$. neoformans. None of the hundred samples showed any growth of $H$. capsulatum. Therefore isolation of $C$. neoformans from bird droppings collected from the study site has provided useful informations for ecological and epidemiological studies of $C$. neoformans which may help to prevent public Health hazards.

\section{Introduction}

Cryptococcosis is an acute, sub-acute or chronic fungal disease caused by an encapsulated basidiomycetous yeast belonging to the genus Cryptococcus (Jagdish Chander). It is among the most prevalent life- threatening mycoses and has a worldwide distribution (Meyer et al., 2003). Among the many species of the genus "Cryptococcus" that are prevalent ubiquitously in environment, C. neoformans and $C$. gattii are 
clinically significant as they are generally viewed to be the most pathogenic species. Both the species are pathogenic to man and animal. Cryptococcal meningitis is considered an AIDS defining condition (Abadi et al., 1999) and it is the most common fungal infection of the central nervous system and the third most frequent neurological complication in AIDS patients (Del Valle, 2006). Approximately 5-13\% of the patients with AIDS suffer from cryptococcal meningitis. Cryptococcosis has been responsible for great morbidity and mortality among patients with AIDS (Fernandez et al., 2003; Rozenbaum et al., 1994; Ruiz et al., 1982) and considered the fourth most common infection in the immunocompromised individuals (Hubalek, 1975). The clinical manifestations of the disease "Cryptococcosis"; caused by various species of encapsulated baisdiomycetous yeasts of genus Cryptococcus are influenced more strongly by host's immune status than by cryptococcal variety. The initial infection most likely occurs through inhalation of infectious airborne small forms of the organism or basidiospores from the environment. The fungal propagules are deposited in the respiratory tract where they may be transient inhabitants or may colonize the mucosa and subsequently disseminate to extrapulmonary sites and present as the cerebrospinal or generalized cryptococcosis. Depending upto the anatomincal sites, cryptococcosis may be: 1. Pulmonary Cryptococcosis 2. CNS Cryptococcosis.3. Visceral Cryptococcosis. 4. Osseous Cryptococcosis. 5. Cutaneous Cryptococcosis.

Histoplasmosis is a granulomatous fungal disease caused by a intracellular dimorphic fungi. Although worldwide in distribution this organism is more prevalent in certain parts of North and central America and has been documented in the soil of the Gangetic plains of India (Sanyal et al., 1975). Growth of the fungus is most frequently associated with soil enriched by excreta of bats, chickens and other birds. The organism has been isolated from bat caves, bird roots, chicken houses and similar enviornments. $H$. capsulatum flourishes in soil fertilized by bird droppings or bat guano. It exists in two forms - the infective mycelial or mould form in the soil and the yeast form in human macrophages. The clinical spectrum of histoplasmosis ranges from asymptomatic infection to progressive disseminated histoplasmosis depending upon the intensity of exposure and the immune status of the exposed individual. Patient's presentation can therefore vary form an acute rapidly fatal course with diffuse interstitial or reticulo-nodular lung infiltrates causing respiratory failure, shock, hepatosplenomegaly and multi-organ failure to a more subacute course with a focal organ distribution involving Liver, Spleen, adrenals, muco-cutaneous regions and bone-marrow. Therefore it is obvious from various studies that there is a strong association of these two pathogenic fungi with bird droppings and soil contaminated with bird dropplings. Though several studies have been conducted at North India regarding presence of Cneoformans in soil and bird excreta and existence of $\mathrm{H}$ capsulatm in the soil of Gangetic delta of West Bengal, no such studies have yet been conducted in North-eastern part of the country. Therefore the present study has been conducted with a view to know the association of $C$. neoformans and $H$. capsulatum with the bird droppings and soil contaminated with bird droppings at Kulik Bird sanctuary, Raiganj, Uttar Dinajpur, West Bengal.

The main aim and objectives of this study includes to assess the presence of these two pathogenic fungi in soil and bird droppings (bird excreta) of the study site. And to find out incidence of any one or both of these two pathogenic fungi. Also to gather useful 
information about ecology and epidemiology of these two fungi for further studies.

\section{Materials and Methods}

The study was carried out in the Microbiology Department of Mata Gujri Memorial Medical College and Lions Seva Kendra Hospital, Kishanganj, Bihar.

Study period was of one year duration from $1^{\text {st }}$ November 2014 to $31^{\text {st }}$ October 2015.

Sample size was 100; of which 50 were birds excreta samples and 50 were soil samples (Soil contaminated with birds excreta). Permission was taken from the authority of Kulik Bird Sanctuary.

Samples were collected in clean sterile plastic bags (Plastic packets of disposable hand gloves) using sterile spatulas and then properly sealed and labeled mentioning the date, type and site of collection. The average sample weight was about $30 \mathrm{gm}$ to $100 \mathrm{gm}$. After collection, samples were carried to the Microbiology laboratory of MGM medical college, Kishanganj, Bihar and were stored in refrigerator (temp $2^{0} \mathrm{C}$ to $8^{0} \mathrm{C}$ ) until used.

Samples were processed according to Casali et al., (2003).

After processing aliquots of $0.5 \mathrm{ml}$ from the supernatant portion of each sample suspension were inoculated on Sabouraud's dextrose agar with chloramphenicol $(200 \mathrm{mg} / \mathrm{L})$ and cycloheximide $(500 \mathrm{mg} / \mathrm{L})$ in duplicate and at the same time the $\mathrm{pH}$ of the solutions were measured. In elaboration from each sample suspension 4 inoculations were done. $1^{\text {st }}$ set: Two on Sabouraud's dextrose agar with chloramphenicol $(200 \mathrm{mg} / \mathrm{L})$ for isolation of Cryptococcus neoformans. $2^{\text {nd }}$ set: Two on Sabouraud's dextrose agar with chloramphenicol $(200 \quad \mathrm{mg} / \mathrm{L}) \quad$ and
Cyclohexamide $(500 \mathrm{mg} / \mathrm{L})$ for isolation of Histoplasma capsulatum.

Then from each duplicated sets of inoculated media: one was incubated at $30^{\circ} \mathrm{C}$ and other was incubated at $37^{\circ} \mathrm{C}$. Cultures those were on SDA with chloramphenicol were incubated up to 15 days. Cultures those were on SDA with Chloramphenicol and Cycloheximide were incubated up to 6 weeks. All the cultures were examined daily during first week and twice a week for rest of the weeks. After appearance of growth, at first the inoculated Sabouraud's dextrose agar media with chloramphenicol that were incubated at $37^{\circ} \mathrm{C}$ followed by the inoculated sabourauds dextrose agar media with chloramphenicol that were incubated at $30^{\circ} \mathrm{C}$ were searched for creamy mucoid/creamy colonies. When such type of colonies were observed, those growth were sub-cultuerd on Sabouraud's dextrose agar media with chloramphenicol to obtain pure colonies. All the isolated colonies (Creamy or Pasty) were identified as yeasts according to colony morphology (Creamy/pasty colony) and microscopic morphology (Budding properties as observed in $\mathrm{KOH}$ and gram stain) of yeast cells. At first India-ink preparation was done to detect capsule of Cneoformans. When no capsule was found the next step done was Germ tube test. Yeast isolates those were germ tube test positive were suspected as Candida albicans growth and was confirmed by microscopic appeareance (budding yeast cells with pseudohyphae) and colony morphology of $\mathrm{C}$. albicansspp and Chlamydospore formation on corn meal agar and growth at $42^{\circ} \mathrm{C}$ to $45^{\circ} \mathrm{C}$. When the germ tube test was negative then inoculum was taken from those germ tube negative yeast growths and was inoculated on corn meal agar and incubated for 2 weeks at $25^{\circ} \mathrm{C}$. Then the incubated cornmeal agar cultures were daily observed for appearance of growth. In almost all cases on corn meal agar Creamy/ pasty colonies appeared in 72 
hours. Then microscopic morphology of the colonies were determined after performing a. Gram stain b. Lactophenol cotton blue stain or $10 \% \mathrm{KOH}$ stain and searched for blastospores, pseudohyphae, arthrospores and chlamydospores according to the flowchart(a) showing identification scheme of commonly encountered yeast isolates. Yeast isolates which showed only blastospores on corn meal agar after microscopical examination were subjected to "Urease test". Rest of the isolates that showed 'Pseudohyphae and chlamydospore combination' were identified according to their macroscopic and microscopic appeareance as Candida albicans, Trichosporon or Geotrichum spp and Non-albicans Candida spp. Respectively. To differentiate between 'Geotricum' and 'Trichosporon' spp again urease test done and for result 'Yeast Identification Chart' was followed. Germ tube negative yeast isolates that were urease positive but showed red colonies were identified as Rhodotorula spp. Yeast isolates those were germ tube test negative and showed blastospores on corn meal agar culture and were urease positive, were inoculated on Bird seed agar media and incubated at $30^{\circ} \mathrm{C}$ for 15 days. The incubated bird seed agar cultures were daily examined for appeareance of brown colonies. When brown colonies were observed, their microscopic morphology were examined and the yeast isolates were identified as $C$. neoformans which was supported by negative growth on Sabouraud's dextrose agar with chloramphenicol and Cycloheximide; as well as creamy growth on SDA with chloramphenicol at $37^{0} \mathrm{C}$.

Usually Cryptococcus neoformans isolates are identified on the basis of I. Creamy mucoid growth on Sabouraud's dextrose agar with chloramphenicol. II. Growth at $37^{0} \mathrm{C}$. III. Capsule on India ink mount. IV. Brown colonies on birds seed agar media. V. No growth on Sabouraud's dextrose agar containing Cycloheximide. VI. Positive urease test. VII. Positive Inositol assimilation test and negative nitrate assimilation test.

When mycelial growths were observed on both Sabouraud's dextrose agar media with chloramphenicol and Sabouraud's dextrose agar media with chloramphenicol and Cycloheximide, search were made to find out white or buff brown colonies at $30^{\circ} \mathrm{C}$ to identify Histoplasma capsulatum. When such type of colonies were observed then their microscopic examination was done by performing lactophenol cotton blue stain or $\mathrm{KOH}$ mount and searched for prominent, thick walled, large tuberculate macro conidia and also for small, broadly elliptical, smooth walled micro conidia which are the characteristics features of mycelial form of Histoplasma capsulatum. No such microscopic features were detected. But after examining all the mycelial growths microscopically using $\mathrm{LCB} / \mathrm{KOH}$ mount, Penicillium spp. and many other filamentous fungi were indentified according to their macroscopic and microscopic appearance.

\section{Results and Discussion}

A total number of 100 samples (50 birds excreta samples and 50 soil samples) were studied during one year period $\left(1^{\text {st }}\right.$ November, 2014 to $31^{\text {st }}$ October, 2015). Out of these 100 samples 3 samples were positive for Cryptococcus neoformans (incidence 3\%). Among these 100 samples (Birds excreta + soil) 50 soil samples showed no incidence of C. neoformans

In this study no capsule was found in all the three $C$. neoformans isolates. Here $C$. neoformans was identified on the basis of: 1 . Creamy growth on Sabouoraud's dextrose agar with chloramphenicol. 2. Growth at $37^{0} \mathrm{C}$. 3. Brown colonies on bird seed agar media. 4. No growth on Sabouraud's dextrose 
agar containing Cyloheximide. 5. Positive urease test. 6. Positive inositol assimilation test 7. Negative nitrate assimilation test.

No growth of $H$. capsulatum was observed in any of the 100 samples (50 bird excreta samples +50 soil samples).

All the samples both birds excreta and soil were dry. $\mathrm{pH}$ of the sample solutions were between 5.5-7, with $\mathrm{pH} 6$ in $72 \%$ cases. In this study besides $C$. neoformans many other spp. of yeast like fungi and filamentous fungi were isolated from the soil and bird excreta samples of the study site. In 99\% cases sample cultures showed positive result for fungal growths within first week following inoculation with maximum in $72-96$ hours.

The present study involves isolation of Cryptococcus spp. and Histoplasma capsulatum from soil and bird droppings of the study site - Kulik Bird Sanctuary at Raiganj, Uttar Dinajpur, West Bengal. The study findings indicate that avian excreta serve as a major environmental source for the opportunistic fungal pathogen, Cryptococcus neoformans. This finding has already been confirmed by many researchers from different regions of the world (Staib, 1989; Granados, 2005). However Histoplasma capsulatum was not isolated in this study in spite of all efforts. The total incidence of Cryptococcus neoformans was 3 out of 50 birds excreta and 50 soil samples totaling 100 samples (3\%). As the birds were not captive there at the study site, so it could be postulated that the birds excreta samples might contain mixture of faeces of all sorts of birds found there. In 2002, Horta et al reported isolation of 17 clinical and 10 environemntal Cryptococcus neoformans cases from pigeon faeces in Rio de Janeiro state of Brazil. (15). In 1998, Yildriran et al isolated 29 cases of $C$. neoformans from 634 samples of pigeon faeces in Turkey. In this isolation, air humidity and being away from sun light were the influential factors.

A suitable $\mathrm{pH}$ of the samples (birds excreta) may also contribute to the occurrence of $C$. neoformans at the study site. The $\mathrm{pH}$ of the birds excreta and soil samples were between 5.5 to 7 with $\mathrm{pH} 6$ in $72 \%$ cases. The $\mathrm{pH}$ of all the three birds excreta samples that showed positive results in favour of $C$. neoformans were 7. Similar type of relationship between $\mathrm{pH}$ of birds excreta and occurrence of $C$. neoformans was observed in works of Julman Cermeno et al., (2006), while working in Bolivar state, Venzuela.

Climate information from meteorological data of Uttar Dinajpur district shows that average temperature of the study site zone ranges from $20^{\circ} \mathrm{C}$ to $30^{\circ} \mathrm{C}$; which maybe favourable for existence of $C$. neoformans in this zone. In a study in Thailan Kuroki et al, (2004) showed that the weather of Thailand was considered appropriate for growth of $C$. neoformans there with a mean temperature of $26^{\circ} \mathrm{C}-30^{\circ} \mathrm{C}$.

Though pigeon excreta is a major environmental source of $C$. neoformans, but is has been isolated from excreta of other birds also like sparrows and parrots and doves which are frequently found at study site (Mukherjee, 2008).

Rosario (2008) reported from Spain that pigeon droppings were not only the reservoir for Cryptococcus and other birds could act as a reservoir for it too. In a study in Iran Soltani et al., (2013) got low frequency of $C$. neoformans; frequency was $2.5 \%$ (i.e. 3 samples were positive for $C$. neoformans out of 120 samples). Contrary to this report, high frequency of $C$. neoformans in pigeon droppings was shown by Xavier et al., (2013) while working in the Tiruchirapalli district of Tamil Nadu, South India. They showed a positively of $63.6 \%$ (i.e. out of 33 birds 
excreta samples collected 21 was positive for $C$. neoformans. They postulated that this high positive result was due to environmental conditions such as heavy plant growths, large amount of pigeon droppings and possible transfer of $C$. neoformans in all the zones by pigeons. All the 3 positive isolates for Cryptococcus neoformans in the present study were obtained from dry old weathered bird droppings which were in accordance with the previous report by Mishra et al., (1981). They showed that fresh pigeon droppings are devoid of incidence of $C$. neoformans. Ruiz et al., (1981) showed that dry old weathered excreta are a favourable substratum since it has fewer bacteria and other organisms; therefore less competition which could help Cryptococcus neoformans to grow. In present study besides Cryptococcus neoformans some spp. of yeast like fungi and some spp. of filamentous fungi were isolated from soil and bird droppings samples of the study site at different time periods. Among yeast like fungi Candida albicans (10\%), Non-albicans Candida spp. (12\%), Rhodotorula spp. (2\%), Trichosporon spp. (3\%) and Geotricum spp. $(2 \%)$ and among filamentous fungi Penicillium spp. (24\%), Aspergillus spp. (15\%), Mucor spp. (9\%), Fusarium spp. (8\%), Alternaria spp. (2\%), Curvularia spp. (4\%), Trichophyton spp. (6\%), were isolated besides C. neoformans (3\%). Costa et al., (2009) also isolated $C$. neoformans, Candida spp., Rhodotorula spp. and Trichosporon spp from pigeon droppings while working in urban areas of Ishfahan. They also confirmed that urban pigeons are a potential source of pathogenic yeasts (Costa et al., 2010).

Table.1 Frequency of yeast and yeast like fungi observed in the present study

\begin{tabular}{|l|l|c|c|c|}
\hline $\begin{array}{l}\text { S1 } \\
\text { No }\end{array}$ & Name of the Organisms & $\begin{array}{c}\text { Total no of } \\
\text { Sample }\end{array}$ & Total observed & Frequency (\%) \\
\hline 1 & Cryoptococcus neoformans & 100 & 3 & 3 \\
\hline 2 & Candida albicans & 100 & 10 & 10 \\
\hline 3 & Non-albicans Candida spp. & 100 & 12 & 12 \\
\hline 4 & Rhodotorula spp. & 100 & 2 & 3 \\
\hline 5 & Trichosporon spp. & 100 & 3 & 2 \\
\hline 6 & Geotricum spp. & 100 & 2 & \\
\hline
\end{tabular}

Table.2 Frequency of filamentous fungi observed in the present study

\begin{tabular}{|l|l|c|c|c|}
\hline Sl No & Name of the Organisms & $\begin{array}{c}\text { Total no of } \\
\text { Sample }\end{array}$ & Total observed & Frequency (\%) \\
\hline 1 & Penicillium spp & 100 & 24 & 24 \\
\hline 2 & Aspergillous spp & 100 & 15 & 15 \\
\hline 3 & Mucor spp & 100 & 9 & 9 \\
\hline 4 & Fusarium spp & 100 & 8 & 2 \\
\hline 5 & Alternaria spp & 100 & 2 & 4 \\
\hline 6 & Curvularia spp & 100 & 4 & 6 \\
\hline 7 & Trichophyton spp & 100 & 6 & 0 \\
\hline 8 & Histoplasma capsulatum & 100 & 0 & \\
\hline
\end{tabular}


Fig.1 Showing the frequency of different yeast and yeast like fungi

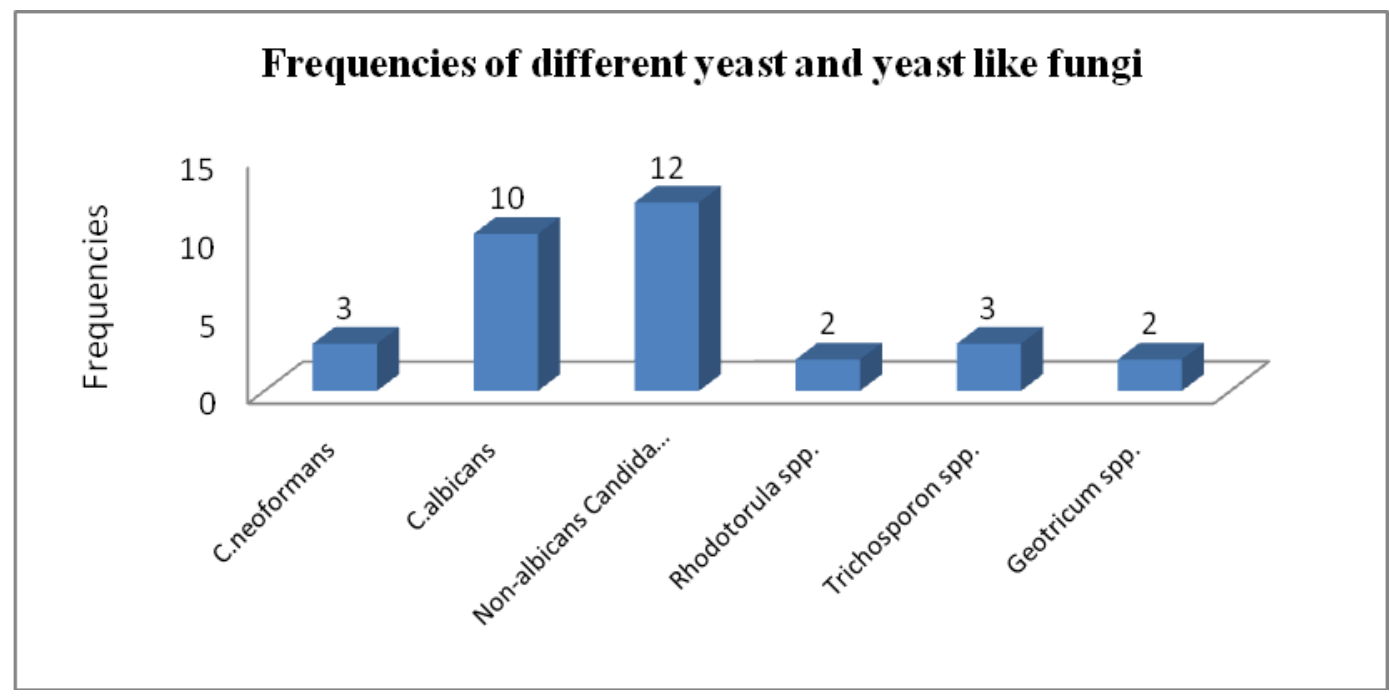

It is observed from table 1 and also from the figure 1 that among yeast and yeast like fungi Nonalbicans Candida spp. showed the highest percentage (12) of frequency followed by Candida albicans (10), Cryoptococcus neoformans (3) Trichosporon spp(3), Rhodotorula spp, (2) and Geotricum $\operatorname{spp}(2)$.

Fig.2 Showing the frequency of different filamentous fungi

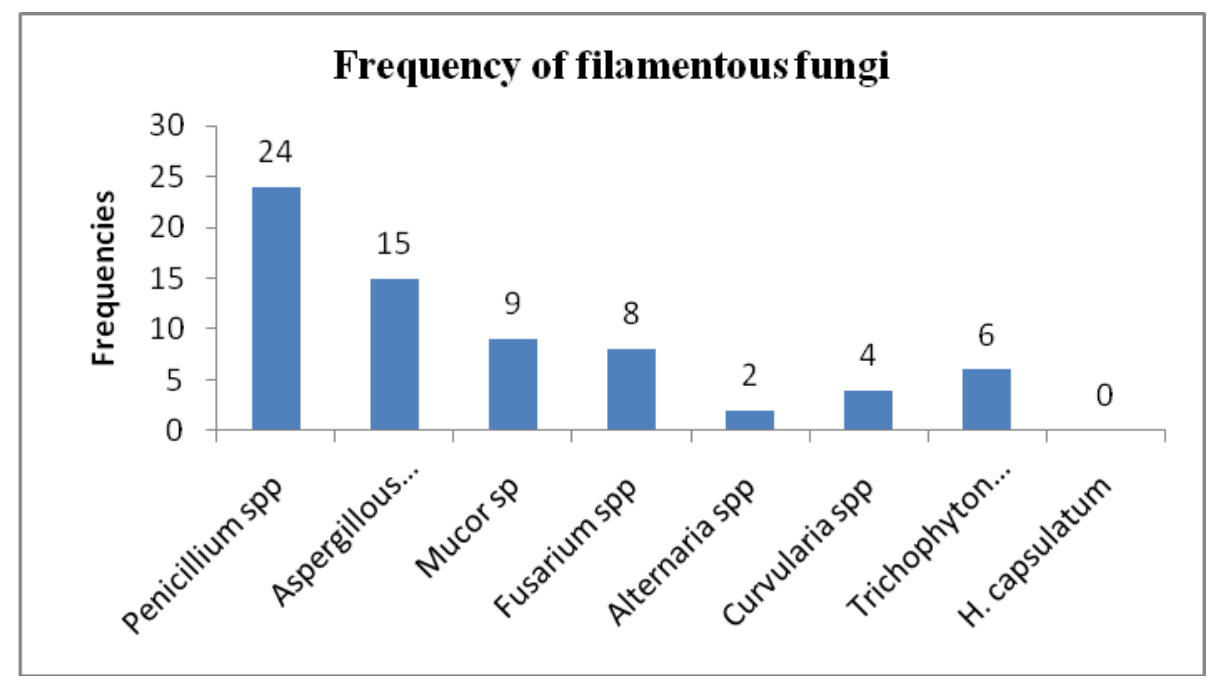

The frequency of filamentous fungi is tabulated in the table 2 and also represented in the figure 2. It is depicted from the table as well as from the figure that Penicillium spp scored the maximum percentage (24) of frequency followed by Aspergillus spp (15), Mucor spp (9), Trichophyton spp, Fusarium spp, Curvularia spp, and Alternaria spp. It is also observed that there was no growth of Histoplasma capsulatum in any sample. 
Picture.1 Shows creamy/ pasty colonies of yeast isolates on SDA

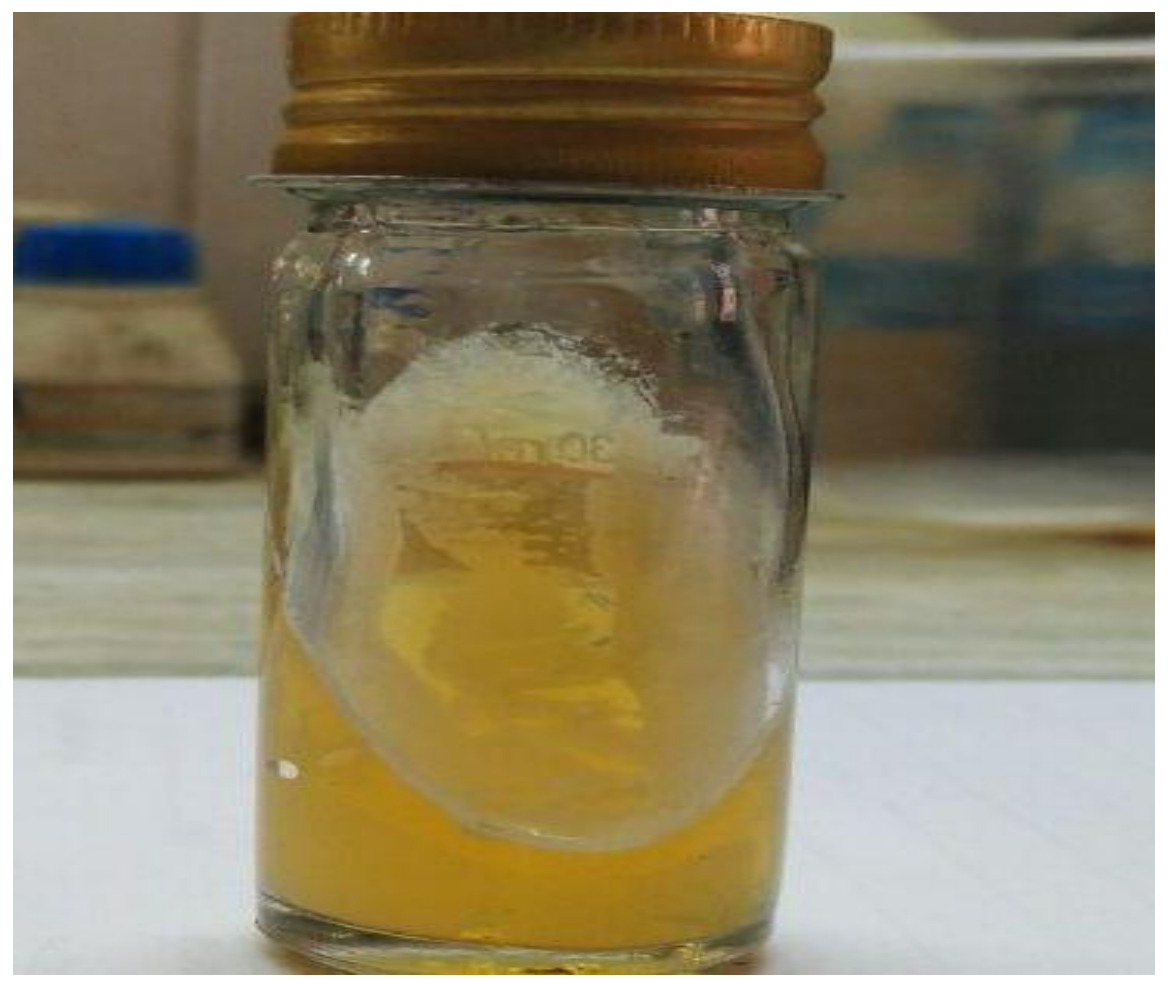

Picture.2 Gram stain showing gram positive round budding yeast cells of $C$. neoformans

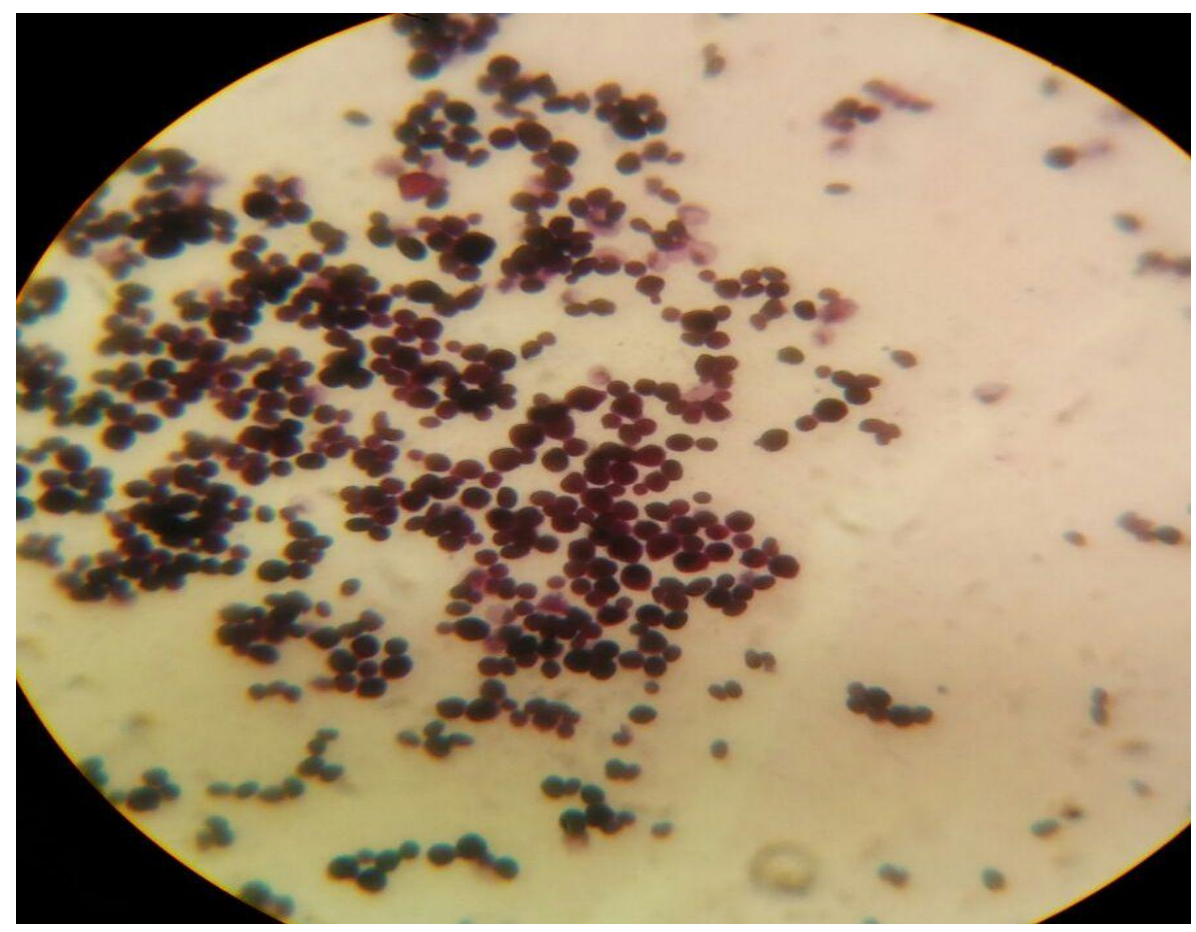


Picture.3 and 4 Budding yeast cells in $\mathrm{KOH}$ mount taken from cornmeal agar culture
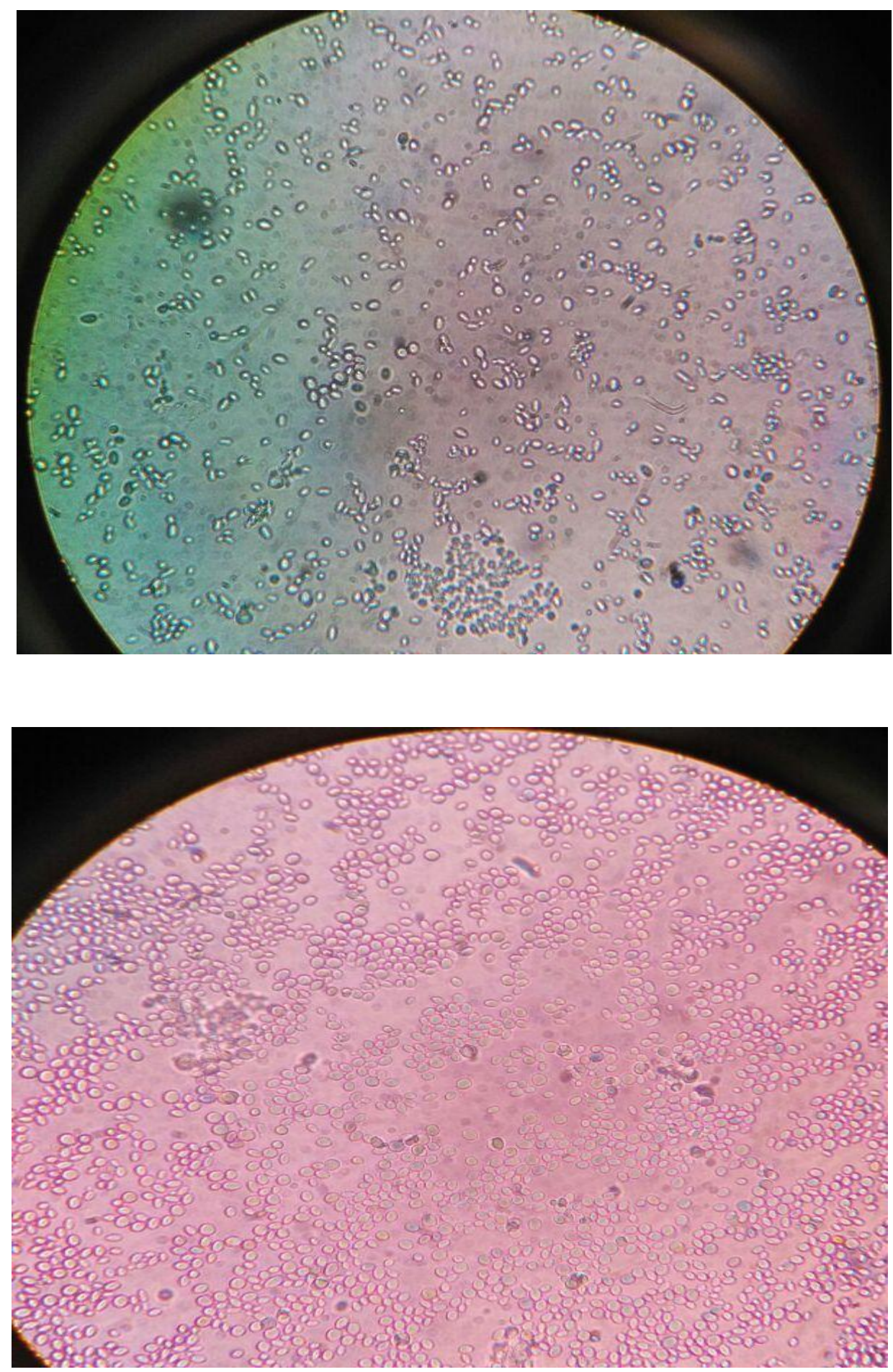
Picture.5 C. neoformans isolates showing urease test positive (3 right side test tubes) left one is un-inoculated urease medium

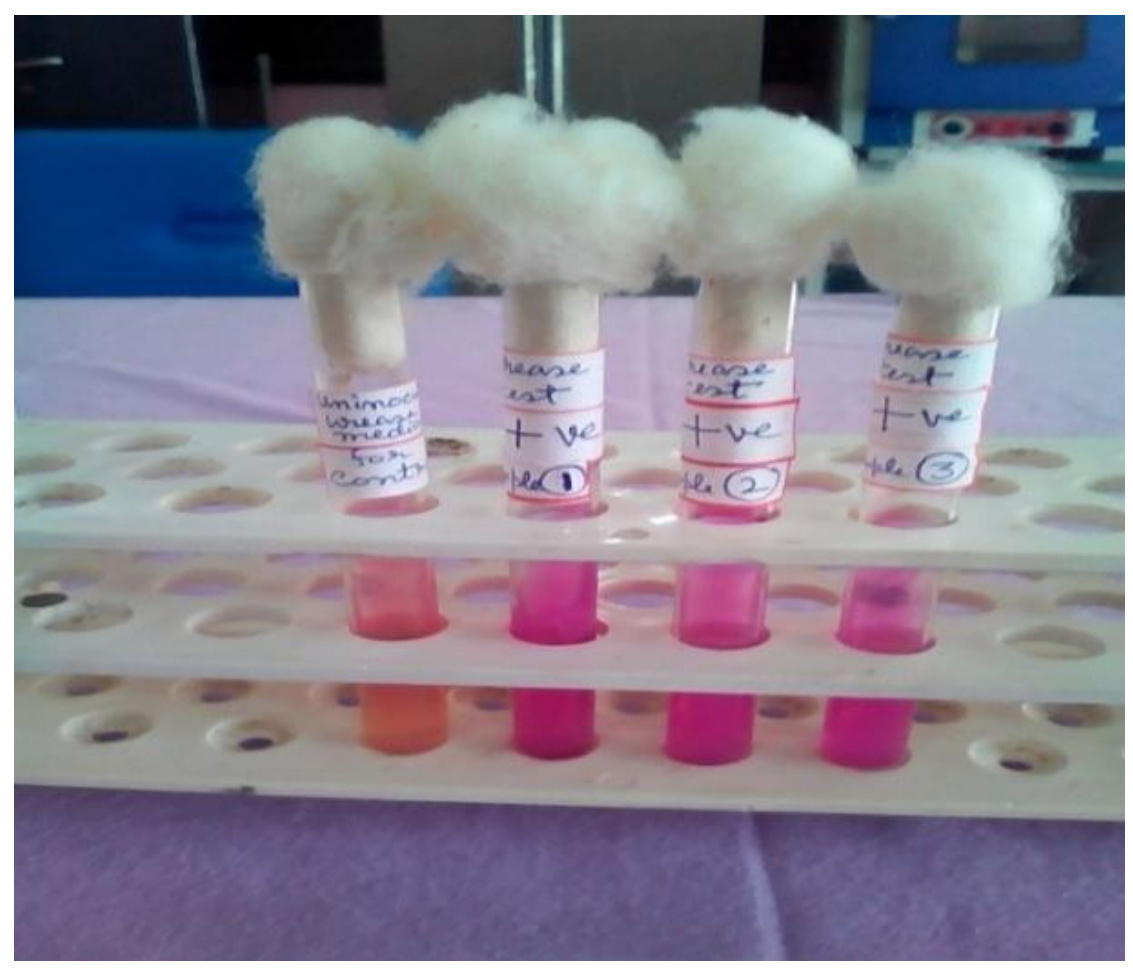

Picture.6 Bird seed agar showing brown colonies of $C$. neoformans

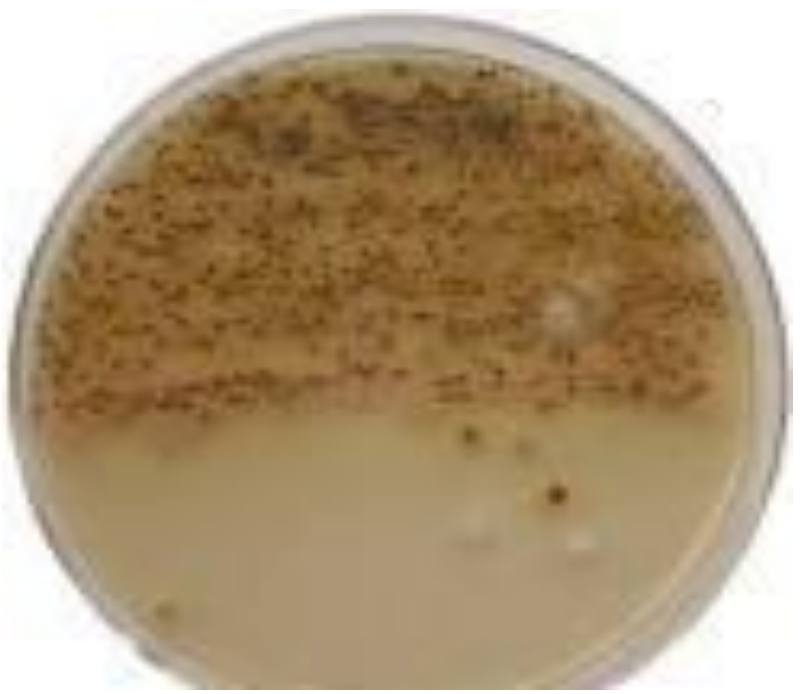

The isolation of saprophytic fungi like Penicillium spp., Aspergillous spp., Mucor spp., Candida spp. from the pigeon droppings was also confirmed by study of Khosrvi (1997) while working in different regions of northern Iran. In this study Histoplasma capsulatum could not be isolated from any of 100 samples comprising of both birds excreta and soil contaminated with birds excreta. In a study in Boliver state, Venezuela, Cermeno et al., (2006) got low incidence (1.3\%) of $H$. capsulatum after examining 116 doves 
excreta samples. According to them the ecology of $H$. capsulatum has been widely studied in the last decade and it has been considered that this fungus is very likely to be present in soils contaminated with bat's guano or birds excreta and therefore it is frequenty isolated from caves, mines, farms, woods, old house or any place where these creatures live. Again in north-east part of India no previous study on occurance and identification of Histoplasma capsulatum was conducted, so that the result of this study can be compared. In this study capsule was not found in $C$. neoformans isolates. This finding correlates with yeast identification chart by Jagdish Chander. In a report on Cryptococcosis on 2013, Institute for International Co-operation in animal Biologics, Iowa, USA, stated that though in the body of a host $C$. neoforamans strains produce polysaccharide capsule around them, in environment they usually proliferate as saprophytic yeasts without any capsule (Cryptococcosis, 2013). The findings in present study indicated that the Kulik bird sanctuary is a potential source for this air borne pathogenic fungi suggesting a public health hazard. Besides, C. neoformans, other fungi like Candida albicans, Non-albicans Candida spp, Rhodotorula spp, Trichosporon spp, Geotricum spp, Penicilium spp, Aspergillous spp, Mucor spp, Fusarium spp, Alternaria spp, Curvularia spp, Trichophyton spp, were isolated from soil and bird droppings of the study site with highest frequency of Penicillium spp (24\%) among filamentous fungi and among yeast and yeast like fungi Non-albicans Candida spp showed highest frequency (12\%). These fungi can be dangerous to immunocompromised patients. Therefore, from this study it is suggested to maintain personal protection measures for the visitors as well as workers of the sanctuary.

\section{References}

Abadi, J., Nachman, S., Kressel, A.B., and Pirofski, L.A. 1999. "Cryptococcosis in children with AIDS," Clinical Infecstious Diseases, vol. view at Google Scholar, 28: no. 2, pp. 309-313.

Casali, A.K., Goulart, L., Rosa, E., Silva, L.K., Ribeiro, A.M., Amaral, A.A., sAlves, S.H. et al. 2003. Molecular typing of clinical and environmental Cryptococcus neoformans isolates in Brazilian state Rio Grande do Sul. FEMS Yeast Res., 3: 405-415.

Cermeno, J., Hernandez, I, Cabello, I., et al. 2006. Cryptococcus neoforamans and Histoplasma capsulatum in dove's (Columbia livia) excreta in Boliver State, Venezuela. Rev. Latinoam. Microbiol., 48: 6-9.

Cermeno, J., Hernandez, I., Cabello, I. et al. Cryptococcus neoforamans and Histoplasma capsulatum in dove's (Columbia livia) excreta in Boliver State, Venezuela. Rev Latinoam Microbiol., 48: 69.

Costa, A.K., J.J. Sidrim, Cordeiro, R.A., Brilhante, R.S., Monteiro, A.J., Rocha, M.F. 2010. Urban pigeons (Columba livia) as a potential source of pathogenic yeasts: A focus on antifungal susceptibility of Cryptococcus strains in Northeast Brazil. Mycopathologia, 169: 207-13.

Cryptococcosis. 2013. (Page No 3 out of 14) by Institute for International Co-operation in Animal Biologics; Iowa state University college of veterinary medicine. Iowa, USA.

Del Valle, L. and Pina-Oviedo, S. 2006. "HIV disorders of the brain; pathology and pathogenesis," Frontiers in Biosci., vol. 11, no. 1, pp. 718-732.

Emmons, C.W. 1995. Saprophytic sources of Cryptococcus neoformans associated with the pigeon (Columba livia). Am. J. Hyg., 62: 227-232.

Fernandez, O.F.L, Costa, T.R., and Costa, M.R. 2000. Cryptococcus neoformansisolados de pacientes com AIDS. Rev. Soc. bras. Med. Trop., 33: $75-78$.

Francis Xavier, T., Auxilia, A., Kannan, M., Freeda Rose, A. and Senthil Kumar, S.R. 2013. Isolation and Identification of Cryptococcus neoformans from pigeon droppings in Tiruchirappalli district of Tamil Nadu, South India, Int. J. Curr. Microbiol. Appl. Sci., ISSN: 2319-7706 
Vol. 2 No. 11 (2013) pp. 404-409.

Granados, D.P., Castaneda, E. 2005. Isolation and characterization of Cryptococcus neoformans varieties recovered from natural sources in Bogota, Columbia and study of ecological conditions in the area. Microb. Ecol., 49: 282-290.

Horta, J.A., Staats, C.C., Casali, A.K., Ribeiro, A.M., Schrank, I.S., Schrank, A., Vainstein, M.H. 2002. Epidemiological aspects of clinical and environmental Cryptococcus neoformans isolates din the Brazilian state Rio Grande do Sul. Med. Mycol., 40(6): 565-71.

Hubalek, Z. 1975. Distribution of Cryptococcusdneoformans in pigeon habitat. Folia Parasit., 22: 73-79.

Jagdish Chander. Textbook of Med. Mycol., (3 ${ }^{\text {rd }}$ Edition).

Khosravi, A.R. 1997. Isolation of Cryptococcus neoformans from pigeon (Columbia livia) droppings in northern Iran. Mycopathologia, 139: 93-5.

Khosravi, A.R. 1997. Isolation of Cryptococcus neoformans from pigeon (Columbia livia) droppings in northern Iran. Mycopathologia, 139: 93-5.

Kuroki, M., Phichaichumpon, C., Yasuoka, A., Chiranairadul, P., Chosa, T., Sirinirund, P., Miyazaki, T., Kakeya, H., Higashiyama, Y., Miyazaki, Y., Ishida, Y., Kohno, S. 2004. Environmental isolation of Cryptococcus neoformans from an endemic region of HIV-associated cryptococcosis in Thailand. 21(10): 809-12.

Li, A., Nishimura, K., Taguchi, H., Tanaka, R., $\mathrm{Wu}$, S., Miyaji, M. 1993. The isolation of
Cryptococcus neoformans from pigeon droppings and serotyping of naturally and clidnically sourced isolates in China. Mycopathologia, 124(1): 1-5.

Meyer, W., Castaneda, A., Jackson, S., et al. 2003. Molecular typing of IberoAmerican Cryptococcus neoformansisolates. Emerg. Infect. Dis., 9: 189-195.

Mishra, S.K., Staib, F., Folkens, U., and Fromtling, R.A. 1981. Serotypes of Cryptococcus neoformans strains isolated in Germany. J. Clin. Microbiol., 14: 106-107.

Mukherjee Arun. 2008. Indian Wildlife Santuaries. Indian Wildlife Portal, 02-05.

Rodsario, I., Acosta, B., and Colom M.F., Pigeons and other birds as a reservoir for Cryptococcus spp. Rev. Iberoam. Micol., 25(1): 513-518.

Rozenbaum, R. and Goncalves, A.J. 1994. Clinical epidemiological study of 171 cases of csruyptococcosis. Clin. Infect. Dis., 18: 369-380.

Ruiz, A., Fromtling, R.A. and Bulmer, G.S. 1981. Distribution of Cryptococcus neoformans in natural site. Infect. Immun., 31: 560-563.

Ruiz, A., Neilson, J.B., and Bulmer, G.S. 1982. Control of Cryptococcus neoformans in nature by Biotic Factors. Sabouraudia, 20: 21-29.

Sanyal, M., Thammayya, A. 1975. Histoplasma capsulatum in the soil of Gangetic plain in India. Indian J. Med. Res., 63: 1020-8.

Staib, F., Hassenkuber, M. 1989. Cryptococcus neoformans in bird droppings: a hygienic epidemiologic challenge. AIDA-kforchung, 12: 649-655.

\section{How to cite this article:}

Nibedita Haldar, Atasi Maji, Debaprasad Pal and Atanu Roy. 2017. Isolation of Cryptococcus spp. and Histoplasma capsulatum from soil and Bird Droppings at Kulik Bird Sanctuary, Raiganj, West Bengal. Int.J.Curr.Microbiol.App.Sci. 6(2): 367-378. doi: http://dx.doi.org/10.20546/ijcmas.2017.602.041 